\title{
The Pulsed-Laser Atom Probe: A Review of Its Development and Initial Applications
}

\author{
Gary L. Kellogg 1 \\ 1. Albuquerque, NM, USA
}

The atom-probe field ion microscope [1], or simply "atom probe", combines the single-atom imaging capability of the field ion microscope [2] with a sensitive time-of-flight mass spectrometer to determine the chemical identity of atoms observed within the image. The process by which surface atoms or adsorbates are removed in the atom probe is known as field evaporation when referring to substrate surface atoms or, more generally, field desorption, which includes both surface atoms and adsorbates. In the original atom probe design, a short-duration, high-voltage, electrical pulse is added to a dc bias voltage to stimulate the field desorption process. The chemical identities of the desorbed ions are measured in a time-of-flight mass spectrometer. The use of high-voltage pulses, however, imposes several limitations on the atom probe as discussed below. These limitations led to the development of a new approach to atom-probe mass spectroscopy, in which, a short duration laser pulse, rather than a high voltage pulse is added to the dc bias voltage to stimulate the field desorption process. Two instruments based on this technique were reported in 1980: the pulsed-laser atom probe (PLAP) [3] and the photoninduced field ionization mass spectrometer [4]. The instruments were similar in concept, but differed in design and the suggested mechanism of desorption. Here, I will focus on the design and development of the PLAP and provide examples of early studies that demonstrated several of the proposed advantages of the pulsed-laser approach [5].

The original PLAP was a modification of an existing imaging atom probe (IAP) [6]. Unlike the probehole atom probe (the commonly used design at the time), the IAP measures the time-of-flight of field desorbed species from the entire imaged area of the emitter surface, rather than a pre-determined location. To determine feasibility of pulsed-laser stimulated field desorption, the IAP was modified to allow a beam from a nitrogen laser to intercept the emitter surface from a direction perpendicular to the emitter axis. The beam entered the vacuum chamber through a sapphire window and was focused to the emitter by a quartz lens on an $x-y-z$ manipulator stage. More details on the design of the instrument and considerations that went into the choice of the laser system are discussed in a review by the author [5].

The primary motivation for the development of the PLAP was its potential for obtaining time-of-flight mass spectra from high-resistivity materials - especially Si. Earlier studies showed that it was possible to obtain field ion microscope images of Si with hydrogen as an imaging gas [7], but attempts to analyze $\mathrm{Si}$ with the atom probe were not successful. The lack of success was attributed to the inability of highresistivity materials to transmit the short-duration, high-voltage pulses required for field desorption. Replacing the high-voltage electrical pulse with a laser pulse in the PLAP led to the first successful time of flight mass spectra from silicon [3]. Early applications of the new capability included studies of the interaction of oxygen [8] and hydrogen [9] with Si surfaces.

Another motivation for the development of the PLAP was to address problems associated with electric field effects in atom probe investigations of surface chemical reactions. For example, molecular adsorbates on the surface may appear as dissociated species in the atom-probe spectra. In a voltagepulsed atom probe, the electric field at the time of desorption is essentially fixed. Tiny increases in the 
electric field lead to catastrophic increases in the desorption rate. In a laser-pulsed atom probe the electric field can be varied over a much wider range and compensated with increases or decreases in the laser power. This capability allows one to explore variations in the desorbed species as a function of electric field. PLAP investigations of hydrogen adsorption on Rh [3] and Mo [10] showed that the hydrogen product distribution changes continuously from predominately $\mathrm{H}^{+}$at electric fields comparable to those in voltage-pulsed atom-probes to predominantly $\mathrm{H}_{2}{ }^{+}$at lower applied fields. These results demonstrated that the problem of field-induced dissociation of molecular adsorbates could be eliminated or significantly reduced by using laser pulses. Additional studies of surface chemical reactions with the PLAP followed $[11,12]$.

The ability to vary the field desorption rate with electric field also led to an investigation of the mechanism for the production of multiply charged ions in field evaporation. Higher charge states (up to $4+$ ) were observed from the very beginning of atom-probe mass spectroscopy, but their presence could not be explained within the context of existing field evaporation models. Quantitative PLAP investigations of the variation of charge state with electric field for W [13] was found to be in good agreement with calculations based on a post-ionization model [14], in which the charge state of the of field evaporated ions are further ionized to a higher charge state after desorption by an electron tunneling process. The general validity of the post-ionization model was subsequently established with similar PLAP measurements of other materials [15].

It was also suggested that the use of laser pulses could improve the mass resolution of the atom probe by eliminating the energy deficits associated with high-voltage electrical pulses. Although it was not possible to demonstrate this advantage with the IAP design (the mass resolution is limited inherently by the short drift distance), Tsong et al. [16] later developed a probe-hole-type atom probe and showed that the use of laser pulses led to a mass resolution three times better than even the state-of-the-art, energycompensated atom-probe [17].

\section{References:}

[1] E.W. Müller, J.A. Panitz, and S.B. McLane, Jr., Rev. Sci. Instrum. 39 (1968) p. 83.

[2] E. W. Müller, J. Appl. Phys. 21 (1956) p. 474.

[3] G. L. Kellogg and T. T. Tsong, J. Appl. Phys. 51 (1980) p. 1184.

[4] W. Drachsel, S. Nishigaki, and J.H. Block, Int. J. Mass Spectrom. Ion Phys. 32 (1980) p. 333.

[5] G. L. Kellogg, J. Phys. E: Sci Instrum. 20 (1987) p. 125.

[6] J. A. Panitz, Progress in Surface Science 8 (1978) p. 219.

[7] A. J. Melmed and R. J. Stein, Surface Sci. 49 (1975) p. 645.

[8] G. L. Kellogg, Appl. Surface Sci. 11/12 (1982) p. 186.

[9] G. L. Kellogg, Phys. Rev. B 28 (1983) p. 1957; G. L. Kellogg, JVST A1 (1983) p. 1125.

[10] G. L. Kellogg, J. Chem. Phys. 74 (1981) p. 1479.

[11] G. L. Kellogg, Surface Sci. 111 (1985) p. 205.

[12] C. F. Ai and T. T. Tsong, Surface Sci. 138 (1984) p. 339.

[13] G. L. Kellogg, Phys. Rev. B 24 (1981) p. 1848.

[14] R. Haydock and D. R. Kingham, Phys. Rev. Lett. 44 (1980) p. 1520.

[15] G. L. Kellogg, Surface Sci. 120 (1982) p. 319.

[16] T. T. Tsong and T. J. Kinkus, Phys. Rev. B 29 (1984) p. 529.

[17] E. W. Muller and S. V. Krishnaswami, Rev. Sci. Instrum. 45 (1974) p.1053. 